\section{Vor der zweiten Teilrevision des KVG}

\author{
E. Taverna
}

Den ehemaligen Ständerat Otto Schoch, geb. 1934, könnte man als Hebamme des neuen KVG bezeichnen, dessen Vorentwurf im Spätherbst 1990 an einer Pressekonferenz im Bundeshaus vorgestellt worden war. Otto Schoch wohnt in Herisau und arbeitet mit sieben Partnern zusammen in der gemeinsamen Anwaltskanzlei im benachbarten St. Gallen. Er war von 1969 bis 1978 Kantonsrat von Appenzell-Ausserrhoden und von 1983 bis 1997 Mitglied des Ständerates, den er 1995/96 präsidierte. Nach dem negativen Volksentscheid über ein neues KVG-Modell 1987 hatte der Bundesrat im Jahre 1989 eine Expertenkommission mit der Ausarbeitung eines neuen Gesetzesentwurfes beauftragt. In dieser Expertenkommission wirkten Vertreter aller interessierten Gruppierungen mit: Ärzte, Zahnärzte, Apotheker, Kantonsvertreter, Krankenkassen, Vertreter von Wirtschaft, Gewerbe und Gewerkschaften usw. Die Kommission, die durch den damaligen Vorsteher des EDI, Bundesrat Flavio Cotti, eingesetzt worden war, erarbeitete zwischen Herbst 1989 und Ende 1990 einen Vorentwurf zu einem neuen KVG, der dem Bundesrat in der Folge als Grundlage für die dem Parlament unterbreitete Gesetzesvorlage diente.

Herr Schoch, wie kamen Sie zu dieser Aufgabe und wie ist sie Ihnen in Erinnerung geblieben?

Ich erinnere mich vor allem daran, dass die Arbeit mit der ungefähr 35 Mitglieder umfassenden Expertenkommission sehr engagiert und intensiv war. Aufgabe der Kommission war es, innerhalb einer Zeitspanne von nur einem Jahr den Entwurf zu einem gänzlich neuen KVG zu konzipieren. Das Geschäft war deshalb so dringlich, weil damals unser ganzes Krankenversicherungssystem zu kollabieren drohte. Denn das alte KUVG aus dem Jahre 1911 war völlig überholt und als Grundlage für ein zeitgemässes soziales Krankenversicherungssystem untauglich geworden. Die Expertenkommission hatte nicht völlig freie Hand, sondern musste Leitplanken respektieren, die ihr durch den Bundesrat gesetzt worden waren. Trotzdem wurden in der Kommission sehr viele Grundsatzfragen eingehend erörtert - um hier nur einige Beispiele zu nennen: Finanzierung der Gesundheitskosten durch Kopfprämien oder über Lohnprozente; Kassenvielfalt oder nur eine einzige Krankenversicherung (ähnlich der SUVA); Beiträge der öffentlichen Hand an die Spitalkosten; System der Prämienverbilligungen; Limitierung der Leistungserbringer usw. Die Diskussionen innerhalb der Kommission waren stets ausserordentlich angeregt, zum Teil geradezu heftig, und gelegentlich musste auch

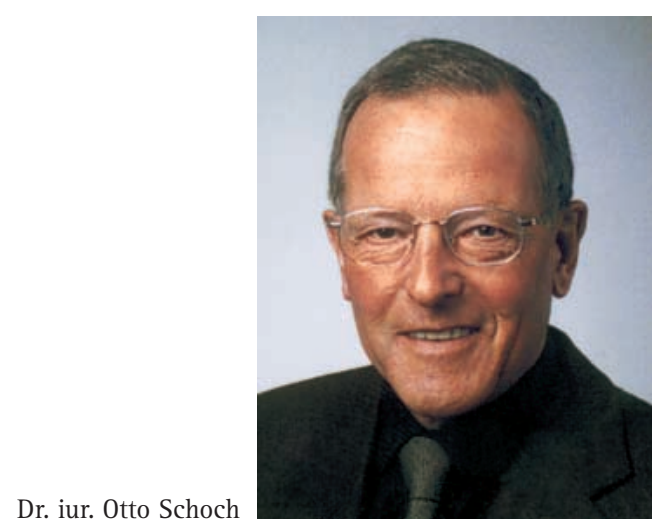

darauf geachtet werden, dass überbordender Eifer einzelner Kommissionsmitglieder nicht zu offenem Streit führte.

\section{Wie ist es nach Abschluss der Kommissionsarbeit} weitergegangen?

Der Bundesrat hat den Gesetzesentwurf der Expertenkommission zunächst seinerseits überarbeitet und die überarbeitete Fassung dann in ein Vernehmlassungsverfahren bei den Kantonen, Parteien, interessierten Verbänden usw. geschickt. Die Ergebnisse dieses Vernehmlassungsverfahrens sind in der Folge bei der Ausarbeitung der Vorlage berücksichtigt worden, die der Bundesrat schliesslich den beiden Kammern des Parlamentes unterbreitet hat. Im Parlament wurde das Geschäft unter grossem Zeitdruck bearbeitet, da es sich mittlerweile als unumgänglich erwiesen hatte, sogar notrechtliche Massnahmen anzuordnen. Der Ständerat hat die bundesrätliche Vorlage, die er als Erstrat behandelte, nur relativ geringfügig verändert. Im Nationalrat wurde der Entwurf des Bundesrates dann aber in mehreren Teilbereichen erheblich umgebaut. Nach durchgeführtem Differenzbereinigungsverfahren passierte das neue Gesetz die Schlussabstimmung in der März-Session 1994. Dabei war allen Beteiligten klar, dass es nun darum gehen musste, Erfahrungen zu sammeln, und gestützt auf diese Erfahrungen, das Gesetz relativ bald schon einer ersten, später allenfalls auch einer zweiten Revision zu unterziehen. Weil gegen die Vorlage das Referendum ergriffen wurde, erhielten Stimmbürgerinnen und Stimmbürger Gelegenheit, darüber abzustimmen. Im Abstimmungskampf habe ich mich für die Annahme des neuen KVG eingesetzt.

\section{Wie beurteilen Sie die Vorschläge}

der zweiten Teilrevision und wie geht es weiter?

Gestützt auf Anträge der durch Ständerätin Christine Beerli präsidierten Kommission für Soziale Sicherheit und Gesundheit hat der Ständerat in der Herbstsession dieses Jahres im wesentlichen drei Massnahmen beschlossen: Die dual-fixe Finanzierung der Spitäler; die Aufhebung des absoluten Kontrahierungszwanges und eine Neuregelung der Prämienverbilligungen in dem Sinne, dass die Prämien 8\% des Familieneinkommens nicht übersteigen dürfen. Ich halte diese Massnahmen für zweckmässig und beurteile deshalb 
die durch den Ständerat gefassten Beschlüsse positiv. Effektiv ging ich selbst von allem Anfang an davon aus, dass Kassen und Kantone je die Hälfte der Kosten für einen Spitalaufenthalt mit Basisservice zu bezahlen hätten, und zwar sowohl für Grundversicherte wie auch für Personen mit Zusatzversicherungen. Auch die Begrenzung des Aufwandes für die soziale Krankenversicherung auf 8\% des Familieneinkommens entspricht Vorschlägen, die seinerzeit bereits in der Expertenkommission diskutiert worden waren. Demgegenüber war die Aufhebung des Kontrahierungszwanges in der Expertenkommission nicht mehrheitsfähig. Ich bin aber der Meinung, dass das, was der Ständerat jetzt beschlossen hat, Sinn macht, und ich würde es daher für zweckmässig halten, wenn sich der Nationalrat diesen Beschlüssen im wesentlichen anschliessen würde.

Werden die neuen Regelungen einen Einfluss auf die Kosten haben?

Das hoffe ich, bin aber über alles gesehen mit Bezug auf die Frage der Kostenentwicklung nach wie vor skeptisch. Am Angebot und an der Qualität der medizinischen Leistungen soll sich ja nichts ändern, und nachdem die Möglichkeiten der Medizin auf der einen Seite stets weiterentwickelt werden, andererseits aber die Erhaltung der Gesundheit als eines der zentralsten und wertvollsten Güter der Menschheit betrachtet wird, muss wohl damit gerechnet werden, dass die Kosten weiterhin in einem nicht unbeträchtlichen Mass ansteigen werden. Ich bedaure, dass im Abstimmungskampf über das neue KVG seinerzeit mit Bezug auf die Kostenentwicklung Erwartungen geweckt wurden, die sich als unrealistisch erwiesen haben. Tatsache ist auch, dass heute die Gesundheit für viele Menschen zu einer "raison d'être" geworden ist. Zur Kostensteigerung beigetragen hat aber auch der grosszügige Ausbau des Leistungskataloges. Ich selbst habe in der Expertenkommission seinerzeit eine Position vertreten, die mir nach wie vor zweckmässig erscheinen würde, nämlich die Beschränkung der obligatorischen Krankenversicherung auf existenzbedrohende Erkrankungen, aber offensichtlich ist diese Vorstellung nicht umsetzbar. Unsere Bevölkerung will einen Versicherungsschutz gegen Gesundheitsrisiken, der wesentlich weiter geht als das, was ich mir hätte vorstellen können.

Was bringt uns die nächste Revision?

Irgendwann wird es zu einer Straffung der Tarifstrukturen kommen müssen mit einem einheitlichen Taxpunktwert oder wenigstens einer klar definierten
Bandbreite für den Taxpunktwert, ähnlich wie in der Zahnmedizin. Denkbar ist auch, dass der Konzentrationsprozess unter den Krankenkassen weitergeht vielleicht wird es früher oder später nur noch zwei, drei oder gar nur noch einen einzigen Versicherer geben. Insgesamt rechne ich aber so oder so mit einem weiteren Anstieg der Gesundheitskosten, mindestens so lange, als uns nicht vordergründigere Probleme intensiv in Anspruch nehmen.

\section{Was würden Sie an einer Feier zum Staatsexamen} jungen Ärztinnen und Ärzten sagen?

Wenn ich daran denke, wie ungnädig mich viele vom KVG Betroffene beurteilen, nur weil ich seinerzeit die Expertenkommission präsidiert habe, dann meine ich, junge Ärztinnen und Ärzte müssten dazu aufgerufen werden, nicht nur ihre eigenen, vor allem wirtschaftlichen Interessen im Auge zu haben und die Situation nicht nur aus dieser subjektiven, eigennützigen Sicht zu beurteilen, sondern sie müssten sich darum bemühen, die Problematik und ihre künftige berufliche Tätigkeit in einem breit abgestützten gesellschaftspolitischen Umfeld zu sehen. Darüber hinaus halte ich aber auch ein von intensiver Kommunikation begleitetes partnerschaftliches Verhältnis zwischen Arzt und Patient für elementar notwendig. Soweit ich selbst als Patient betroffen bin, habe ich diesbezüglich eigentlich stets nur gute Erfahrungen gemacht - immerhin ein Lichtblick!

\section{Sind Sie nach Ihrer politischen Tätigkeit immer} noch öffentlich aktiv?

Neben meiner beruflichen Tätigkeit als Rechtsanwalt, der ich mich in erster Linie widme, bin ich seit Anfang 1998 Ombudsmann von Schweizer Radio und Schweizer Fernsehen DRS. In dieser Eigenschaft hatte ich im vergangenen Jahr insgesamt 268 Reklamationen von Radiohörerinnen und -hörern und Fernsehzuschauerinnen und -zuschauern zu bearbeiten. Dabei ging es unter anderem auch um Reklamationen von Zahnärzten, mit denen sich die Sendung Kassensturz des Schweizer Fernsehens beschäftigt hatte. Beanstandungen von Ärzten wegen Fernseh- oder Radiosendungen hatte ich demgegenüber nie zu bearbeiten. Ich bin überdies Präsident einer Stiftung des Schweizerischen Roten Kreuzes, die humanitäre Zielsetzungen verfolgt und deren Führung mich in den letzten zwei Jahren intensiv in Anspruch genommen hat.

Herr Schoch, ich danke Ihnen für dieses Gespräch und wünsche Ihnen gute Gesundheit. 